\title{
Hysteresis Properties of Corroded High Strength Steel
}

\author{
Hong Wang ${ }^{1 *}$ \\ ${ }^{1}$ Weifang Branch of Delin United Engineering Co., Ltd, Wei Fang, China
}

\begin{abstract}
In order to study the effects of corrosion damage on the hysteresis properties of high strength steel (HSS), dry-wet cycle corrosion tests were conducted on Q690D steel to obtain 6 batches of corroded specimens, and cyclic loading tests were performed on them to analyse the degradation law of hysteresis curve, skeleton curve and hysteretic energy, and then establish the hysteresis constitutive model of corroded HSS steel. Results indicated that although the hysteresis curves of corroded specimens are still smooth and full, corrosion damage would result in the continuous decrease of peak stress, and lead to the linear reduction of hysteresis energy. Besides, the cyclic hardening coefficient $K$ ' and cyclic hardening index $n$ ' of HSS would also decrease linearly with corrosion degree. Based on these experimental results, a new hysteresis constitutive model for corroded HSS steel was proposed, and its validity was verified.
\end{abstract}

\section{Introduction}

The use of high strength steel (HSS) in steel structures can effectively reduce member cross-sections, thereby reducing costs. However, steel structures under corrosive environment for a long time will inevitably suffer serious corrosion damage. Corrosion will change surface feature and mechanical properties of steel, resulting in the decrease of strength and deformability, and even lead to the safety problem of structure. Another non-negligible issue is that the existing corroded steel structures may be threatened by earthquakes during the entire life cycle. Under the earthquake loads, steel structures may undergo a great accumulation of plastic strain and fail after tens of cycles, exhibiting an extremely low cycle damage process with high strain ${ }^{[1]}$.

In recent years, the corrosion mechanism of steel and structural reliability assessment of corroded steel structures have received extensive attention, particularly the monotonic properties ${ }^{[2-4]}$. Compared to the monotonic load, the stress state of corroded steel under low cycle hysteresis loading will change greatly ${ }^{[5]}$. The synergistic effect of cyclic loading damage and steel corrosion damage under earthquakes will aggravate the deterioration of material properties. Bruneau and Zahrai ${ }^{[6]}$ conducted a bending hysteresis performance test on a plate specimen extracted from a naturally corroded $\mathrm{H}$-shaped steel member. The results showed a significantly advanced fracture of the corroded specimen and a significant reduction of cycling number. The consistent conclusions were also drawn by the low-cycle fatigue tests of corroded S500s steel bars carried out by Apostolopoulos [7].

The above researches clearly show that corrosion has a significant degradation effect on the cyclic loading performance of steel, but the research objects are all lowstrength steels. It is well known that the mechanical properties of HSS are quite different from ordinary steels, therefore, the obtained conclusions would not have general applicability. Moreover, the above researches did not give a quantitative description of the hysteretic constitutive model. Therefore, it is necessary to study the hysteretic performance of corroded HSS.

\section{Experimental procedure}

\subsection{Corrosion tests}

The test material in this paper was Q690D steel sheet with dimensions of $280 \times 60 \mathrm{~mm}$ and the thickness was 10 $\mathrm{mm}$. The chemical composition of Q690D steel is listed in Table 1.

The test methods of wet-dry cycle was used for corrosion. First, the specimens were soaked with saturated $\mathrm{NaCl}$ solution ( $\mathrm{pH}$ value 6.0-7.0) for 6 hours. Then the specimens were put into a test box with temperature of $35{ }^{\circ} \mathrm{C}$ and humidity of $93 \% \pm 3 \%$ for 12 hours. Finally, specimens were sequentially removed out for drying treatment. The above operations were repeated every 2 days. After corroded for 0d, 20d, 40d, 60d, 80d and 100d, one specimen was removed out and numbered as $\mathrm{C} 0, \mathrm{C} 1$, $\mathrm{C} 2, \mathrm{C} 3, \mathrm{C} 4, \mathrm{C} 5$, respectively.

Table 1. Chemical composition of Q690D steel (wt \%).

\begin{tabular}{|c|c|c|c|c|c|c|c|}
\hline $\mathrm{C}$ & $\mathrm{Mn}$ & $\mathrm{Si}$ & $\mathrm{P}$ & $\mathrm{S}$ & $\mathrm{Cr}$ & $\mathrm{Mo}$ & $\mathrm{V}$ \\
\hline 0.07 & 1.61 & 0.15 & 0.007 & 0.002 & 0.01 & 0.002 & 0.003 \\
\hline
\end{tabular}

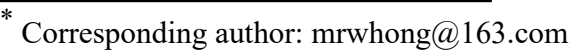




\subsection{Cyclic loading tests}

The geometry of dog-bone specimens for cyclic loading tests designed in accordance with ASTM E466 is schematically shown in Fig. 1. All specimens were tested according to GB/T3075-2008 at room temperature in laboratory air. The cyclic loading tests were carried out in an HT-9711 electro-hydraulic test machine, as shown in Fig. 2. The cyclic tests were performed with an increasing sinusoidal load amplitude with the strain increment of $1 \%$, and the specimens were loaded twice at each strain amplitude (see Fig. 2).

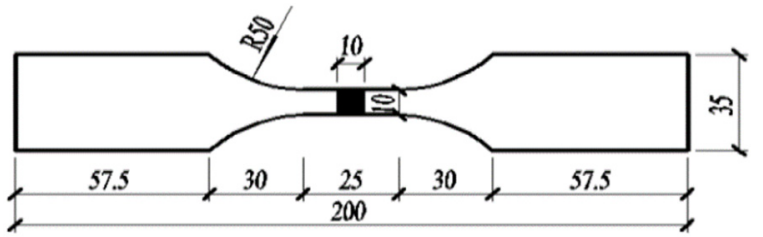

Fig. 1. Dimensions of test specimens for cyclic loading
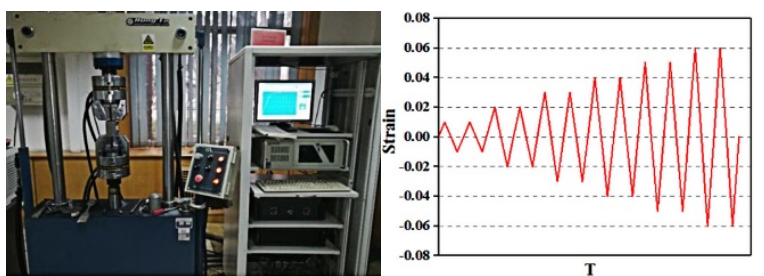

Fig. 2. Loading system

\section{Results and discussion}

\subsection{Corrosion phenomenon}

It can be seen from Fig. 3 that, the surface of non-corroded specimen has a good metallic lustre. At the initial stage of corrosion, the surface of specimen was locally corroded. As the corrosion time increased, the rust layer covered entire surface and was accompanied by formation of yellow-brown corrosion particles. At the late stage of corrosion, a large number of red-brown loose-shaped corrosion products were produced on surface of specimens, which were distributed in flakes and exfoliated locally.
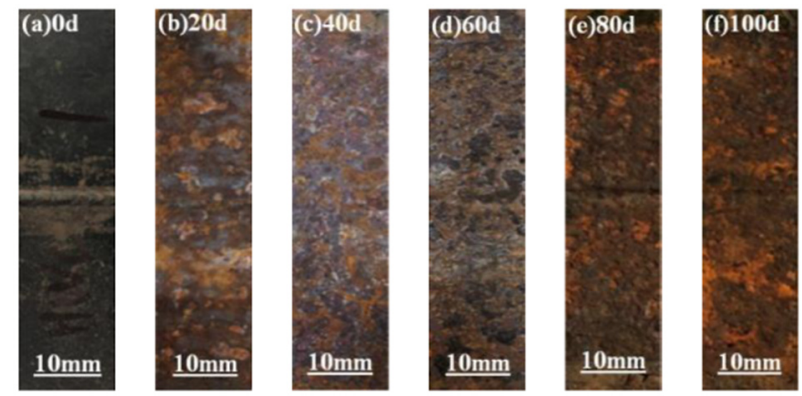

Fig. 3. Corrosion features
The hysteresis curve can reflect the characteristics of strength, stiffness and energy dissipation of material during cyclic loading, and is also the basis of determining the material cyclic constitutive model. Fig. 4 gives the hysteresis curves of all specimens. It can be seen that the hysteresis curves of specimens with different corrosion cycles are smooth and full, without obvious pinch-in phenomenon, indicating that corroded HSS still possess good plastic deformation and energy dissipation capabilities. As the corrosion degree increases, the depth and size of pits on the surface of specimen continue to increase, and stress concentrations are easily formed. Therefore, the peak stress and area of the hysteresis loops decreased continuously after corrosion.

For HSS, there is no obvious yield platform. At the first loading stage, stress change linearly firstly and then nonlinear hardening occurs with the increase of strain. The slope of unloading curve is basically the same as that of elastic stage of first loading. During reloading, the yield limit is reduced and the Bauschinger effect is clearly observed. Two hysteresis loops under the same strain amplitude are not completely coincident, and the latter cycle is larger than former cycle. The main reason is that the yield surface gradually increases with the increase of plastic strain, and the isotropic hardening phenomenon occurs.

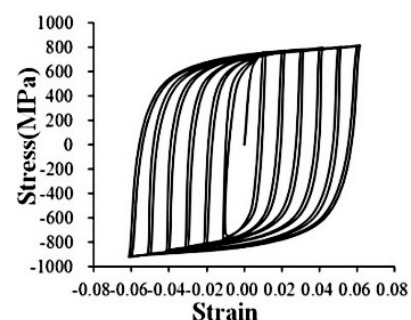

(a) $\mathrm{C} 0,0 \mathrm{~d}$

(c) $\mathrm{C} 2,40 \mathrm{~d}$

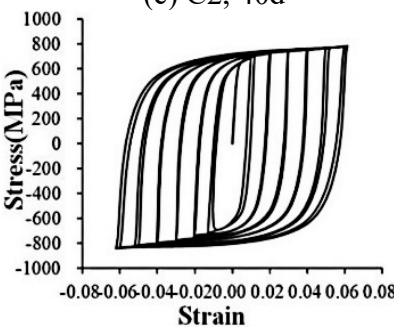

(e) $\mathrm{C} 4,80 \mathrm{~d}$

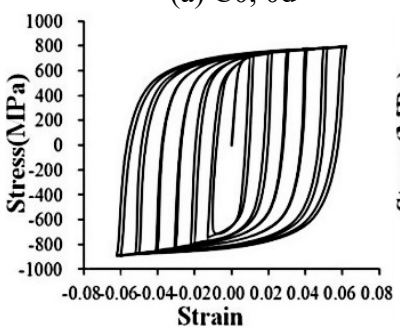

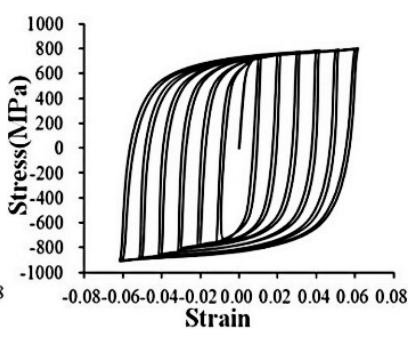

(b) $\mathrm{C} 1,20 \mathrm{~d}$

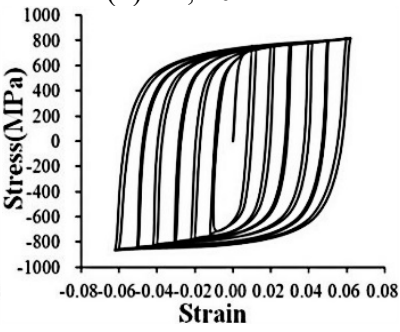

(d) $\mathrm{C} 3,60 \mathrm{~d}$

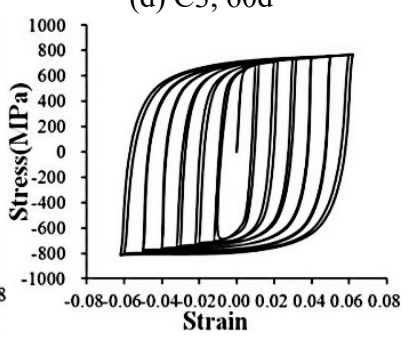

(f) $\mathrm{C} 5,100 \mathrm{~d}$
Fig. 4. Hysteresis curves

\subsubsection{Energy dissipation}

Hysteretic energy can reflect the seismic performance of structure, and it can also reflect the energy dissipation

\subsubsection{Hysteresis curve}


capacity of structure under cyclic loading. The amount of hysteretic energy can be expressed by the area of hysteretic loop. From the data in Fig. 5, it can be seen that although the number of hysteresis cycles of all specimens is the same, but the hysteresis energy $(J)$ shows a linear decreasing trend with the increase of corrosion time $(t)$ :

$$
J=-0.8788 t+856.33
$$

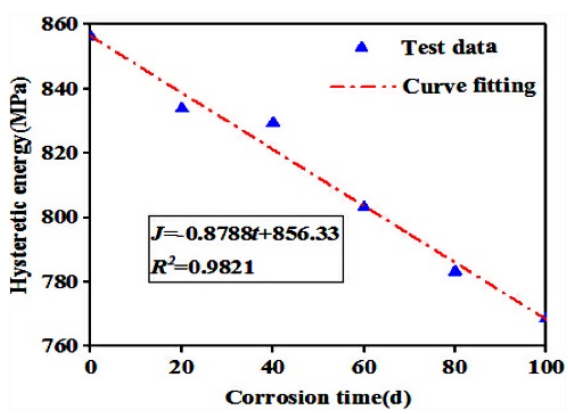

Fig. 5. Degradation law of hysteretic energy

\subsubsection{Skeleton curve}

Fig. 6 shows the skeleton curves of specimens with different corrosion ages. It can be seen that the skeleton curves of Q690 steel under cyclic loading basically has no yield platform. The ultimate strength of steel is reduced because of corrosion. For example, the ultimate strength of specimens decreases by $12.8 \%$ after 100 days of corrosion. Because the presence of rust pits on the surface will cause local stress/strain concentration and redistribution of stress/strain on the surface and inside the steel, which, in turn, will cause further cyclic hardening behaviour.

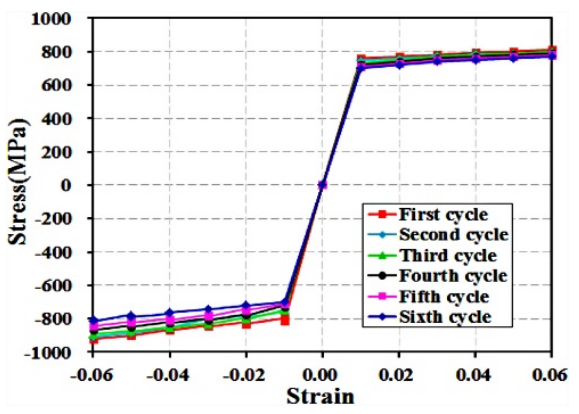

Fig. 6. Skeleton curves

\subsection{Hysteresis constitutive model}

The hysteresis constitutive model consists of four parts, namely the first loading criterion, unloading criterion, cyclic skeleton criterion and reloading criterion ${ }^{[9]}$. The first-time loading curve is consistent with that of monotonic loading, and it is in a state of monotonic tension or compression. The unloading stage is a linear elastic segment, and the unloading slop remains the same as elastic modulus $E_{\mathrm{s}}$ based on the experimental results. Therefore, only the mathematical models of the skeleton curve and reload curve of corroded HSS remain to be proposed.

\subsubsection{Skeleton curve model}

The Ramberg-Osgood model has good applicability to the analysis of hysteretic behaviour of HSS ${ }^{[8]}$, and the mathematical expression is shown in Eq. (2).

$$
\frac{\Delta \varepsilon}{2}=\frac{\Delta \varepsilon_{\mathrm{e}}}{2}+\frac{\Delta \varepsilon_{\mathrm{p}}}{2}=\frac{\Delta \sigma}{2 E_{\mathrm{s}}}+\left(\frac{\Delta \sigma}{2 K^{\prime}}\right)^{\frac{1}{n^{\prime}}}
$$

where $\Delta \varepsilon$ is total strain amplitude, $\Delta \varepsilon_{\mathrm{e}}$ is elastic strain amplitude, $\Delta \varepsilon_{\mathrm{p}}$ is plastic strain amplitude, $\Delta \sigma$ is stress amplitude, $n^{\prime}$ is cyclic hardening index, $K^{\prime}$ is cyclic hardening coefficient, $E_{\mathrm{s}}$ is elastic modulus.

Eq. (2) is used to fit the skeleton curves, and the model parameters are obtained as shown in Fig. 7. It can be seen that cyclic hardening coefficient $K$ ' and cyclic hardening index $n$ ' of HSS decrease linearly with corrosion time, as shown in Eq. (3)-(4). For specimens after 100 days of corrosion, cyclic hardening coefficient and cyclic hardening index decrease by $13.4 \%$ and $37.3 \%$, respectively.

$$
\begin{aligned}
K^{\prime} & =-1.3825 t+983.04 \\
n^{\prime} & =-0.0002 t+0.052
\end{aligned}
$$

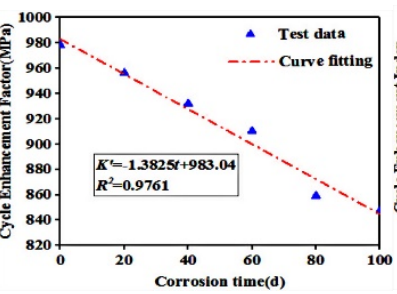

(a) $K^{\prime}$

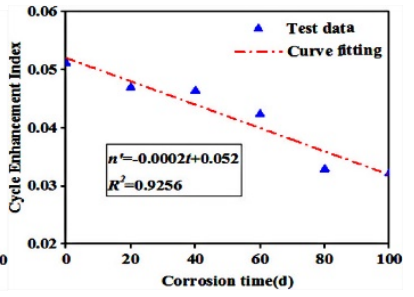

(b) $n$
Fig. 7. Degradation law of model parameters of skeleton curve

\subsubsection{Reloading curve model}

The reloading process $(\mathrm{AB})$ is shown in Fig. 8. The reloading curves points to the historical peak point, and the slope of reloading section is between the secant slope $E_{\mathrm{k}}$ (starting point A to end point B) and unloading stiffness $E_{\mathrm{s}}$. Point $\mathrm{C}$ in this figure is the end point of reloading curve, and also the starting point of unloading curve CA in the compression direction. Assuming that $d$ section in Fig. 8 is equal to $d_{2}+d_{3}$, and the proportion $\eta=d_{3} / d$, then the stress at point $\mathrm{G}$ can be obtained by:

$$
\left\{\begin{array}{l}
\sigma=E_{\mathrm{s}}\left(\varepsilon-\varepsilon_{\mathrm{A}}\right)+\sigma_{\mathrm{A}}-\eta\left(E_{\mathrm{s}}-E_{\mathrm{k}}\right)\left(\varepsilon-\varepsilon_{\mathrm{A}}\right) \\
E_{\mathrm{k}}=\frac{\sigma_{\mathrm{B}}-\sigma_{\mathrm{A}}}{\varepsilon_{\mathrm{B}}-\varepsilon_{\mathrm{A}}}
\end{array}\right.
$$




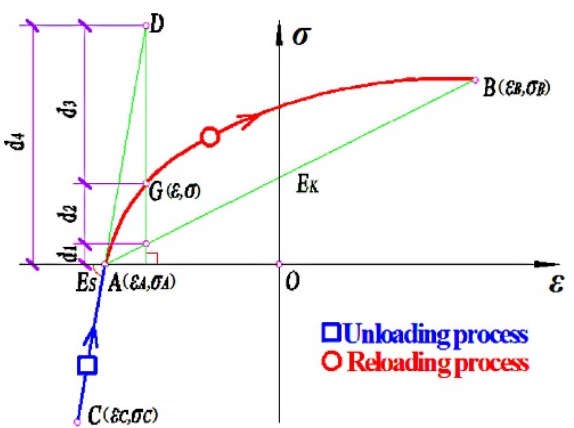

Fig. 8. Reloading curve model

\subsubsection{Hysteresis criterion}

To summary the above four stages of criteria, the specific hysteresis criteria is described as Fig. 9. It is assumed that the stress and strain of the starting point $\mathrm{x}$ on the $k$-th tensile/compression reloading curve is denoted as $\mathrm{x}_{k}\left(\varepsilon_{0}{ }^{\mathrm{t}} \mathrm{c}\right.$, $\left.\sigma_{0}{ }^{t / c}\right)$, and the end point $\mathrm{x}$ on the reloading curve or the starting point $\mathrm{x}$ of the unloading curve is denoted as $\mathrm{x}_{k}\left(\varepsilon_{\mathrm{u}}^{\mathrm{t}} \mathrm{c}\right.$, \left.${\sigma_{\mathrm{u}}}^{\mathrm{t}}\right)$. The initial value of target point is taken as the yield point. When it begins to load, the loading curve points to yield point $\mathrm{y}_{\mathrm{t}}$ along a straight line with a slope of $E_{\mathrm{s}}$. After reaching point $\mathrm{y}_{\mathrm{t}}$, it starts to enter the hardening stage. After reaching the target point A1, the target point of the next tensile reloading cycle is extended to A1 according to the cyclic skeleton rule. Then unloading is started along a straight line with the slop of $E_{\mathrm{s}}$ until to point O1. The compression reloading curve first reaches the yield point and then extends along the compression skeleton curve to point B1. After the compression unloading section, the tensile reloading curve of the second cycle will first reach the target point A1 and then extends along the tensile skeleton curve to point A1.

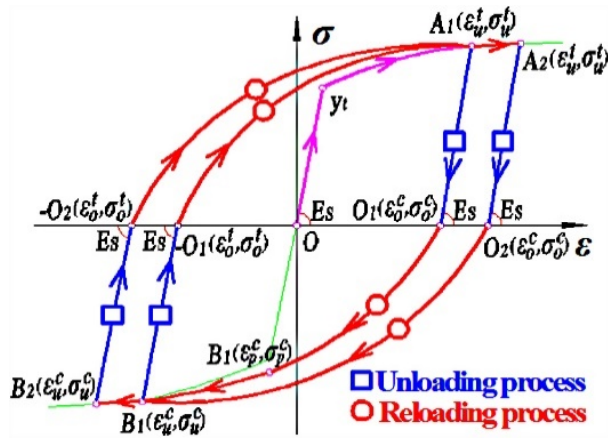

Fig. 9. Hysteresis criteria expression

\subsubsection{Validation}

The comparison between the experimental hysteresis curve and theoretical model is shown in Fig. 10. It can be seen that the theoretical model agrees well with the test results. The given cyclic constitutive model has good applicability to describe the hysteresis performance of HSS with corrosion damage.

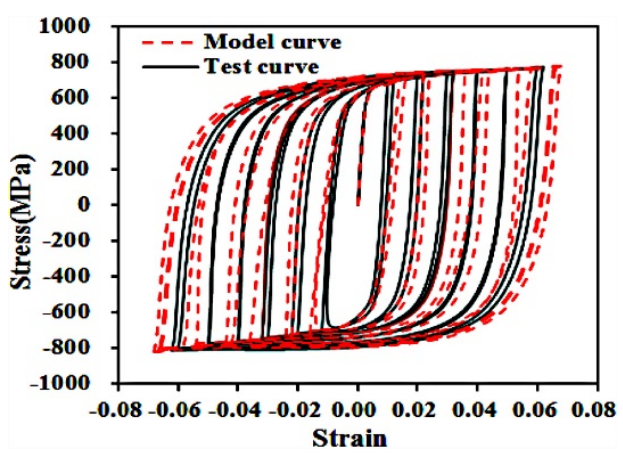

Fig. 10. Comparison between experimental curve and theoretical model

\section{Conclusion}

In this paper, cyclic loading tests are performed on the corroded Q690D steel. The relationship between hysteresis properties and corrosion degree is analysed and a new hysteresis constitutive model of corroded HSS is established. The following conclusions are obtained.

(1) Under cyclic loading, corroded Q690D steel shows obvious cyclic hardening phenomenon and Bauschinger effect. The hysteresis curves of specimens with different corrosion cycles are smooth and full, without obvious pinch-in phenomenon. As the corrosion degree increases, the peak stress and area of the hysteresis loops decreased continuously after corrosion.

(2) Ramberg-Osgood model could well describe the cyclic skeleton curve of corroded Q690D steel. The cyclic hardening coefficient $K$ ' and cyclic hardening index $n$ ' of HSS decrease linearly with corrosion time.

(3) The proposed hysteresis constitutive model agrees well with the experimental hysteresis curves of corroded Q690D steel.

\section{References}

1. T. R. Witcher. Civil Engineering Magazine Archive, 87 (2017).

2. W. Shi, L. Tong, Y. Chen, Z. Li, K. Shen. Journal of Building Structures, 33 (2012).

3. J. K. Paik, J. M. Lee, J. K. Man. Thin-Walled Structures, 42 (2004).

4. T. Nakai, H. Matsushita, N. Yamamoto, H. Arai. Marine Structures, 17 (2004).

5. A. M. Kanvinde, G. G. Deierlein. Journal of Structural Engineering, 132 (2006).

6. M. Bruneau, S. M. Zahrai. Journal of Structural Engineering, 123 (1997).

7. C.A. Apostolopoulos. Construction and Building Materials, 21 (2007).

8. Ramberg W, Osgood W R. Technical Note, 902 (1943).

9. M. Wang, Y. Shi, Y. Wang. Journal of Constructional Steel Research, 79 (2012). 